\title{
Improving aqueous solubility and antitumor effects by nanosized gambogic acid-mPEG 2000 micelles
}

This article was published in the following Dove Press journal:

International Journal of Nanomedicine

27 December 2013

Number of times this article has been viewed

\section{Lulu Cai ${ }^{1, *}$ \\ Neng Qiur,** \\ Mingli Xiang ${ }^{3, *}$ \\ Rongsheng Tong' \\ Junfeng Yan' \\ Lin $\mathrm{He}^{1}$ \\ Jianyou Shi' \\ Tao Chen ${ }^{4}$ \\ Jiaolin Wen ${ }^{3}$ \\ Wenwen Wang ${ }^{3}$ \\ Lijuan Chen ${ }^{3}$}

'Department of Pharmacy, Sichuan Academy of Medical Science and

Sichuan Provincial People's Hospital,

${ }^{2}$ College of Materials and Chemistry

and Chemical Engineering, Chengdu

University of Technology, ${ }^{3}$ State

Key Laboratory of Biotherapy, West

China Hospital, West China Medical

School, Sichuan University, Chengdu,

People's Republic of China; ${ }^{4}$ Faculty of

Pharmacy, University of Montreal,

Montreal, QC, Canada

*These authors contributed equally to this paper

Correspondence: Lijuan Chen

State Key Laboratory of Biotherapy,

West China Hospital, Sichuan University,

4 Keyuan Road, I Gaopeng Street,

Chengdu 6I004I, Sichuan, People's

Republic of China

$\mathrm{Tel}+862885164103$

Fax +8628 85164060

Email lijuan50I@hotmail.com

Lulu Cai

Department of Pharmacy, Sichuan

Academy of Medical Science and

Sichuan Provincial People's Hospital,

Chengdu 6I007I, Sichuan, People's

Republic of China

Tel +862887393316

Fax +862885164060

Email Izxlulu@I26.com
Abstract: The clinical application of gambogic acid, a natural component with promising antitumor activity, is limited due to its extremely poor aqueous solubility, short half-life in blood, and severe systemic toxicity. To solve these problems, an amphiphilic polymer-drug conjugate was prepared by attachment of low molecular weight (ie, $2 \mathrm{kDa}$ ) methoxy poly(ethylene glycol) methyl ether (mPEG) to gambogic acid $\left(\mathrm{GA}-\mathrm{mPEG}_{2000}\right)$ through an ester linkage and characterized by ${ }^{1} \mathrm{H}$ nuclear magnetic resonance. The GA-mPEG ${ }_{2000}$ conjugates self-assembled to form nanosized micelles, with mean diameters of less than $50 \mathrm{~nm}$, and a very narrow particle size distribution. The properties of the GA- $\mathrm{PPEG}_{2000}$ micelles, including morphology, stability, molecular modeling, and drug release profile, were evaluated. MTT (3-(4,5-dimethylthiazo 1-2-yl)-2,5 diphenyl tetrazolium bromide) tests demonstrated that the GA-mPEG ${ }_{2000}$ micelle formulation had obvious cytotoxicity to tumor cells and human umbilical vein endothelial cells. Further, GA-mPEG ${ }_{2000}$ micelles were effective in inhibiting tumor growth and prolonged survival in subcutaneous B16-F10 and C26 tumor models. Our findings suggest that GA-mPEG ${ }_{2000}$ micelles may have promising applications in tumor therapy.

Keywords: gambogic acid, poly(ethylene glycol)-drug conjugate, micelle, antitumor, toxicity

\section{Introduction}

Gambogic acid, the major active ingredient of gamboge, a brownish to orange resin exuded from the Garcinia hanburyi tree in Southeast Asia, possesses significant anticancer activity both in vitro and in vivo. ${ }^{1-4}$ Multiple mechanisms may be involved in its potent antitumor effect, including induction of apoptosis, cell cycle arrest, telomerase inhibition, antiangiogenesis activity, and an antimetastasis effect. ${ }^{5-10}$ Unfortunately, gambogic acid has poor solubility (less than $0.5 \mu \mathrm{g} / \mathrm{mL}$ ), a short half-life (less than 1 hour in dogs and less than 20 minutes in rats), and low bioavailability, which limits its application. ${ }^{11,12}$ Solubilizers such as Cremophor ${ }^{\circledR}$ EL (polyethoxylated castor oil, Sigma-Aldrich, St Louis, MO, USA) and L-arginine have been introduced to solve these problems. ${ }^{11-14}$ However, these agents may cause a series of side effects, such as vascular stimulation, hemolytic toxicity, hypersensitivity reactions, nephrotoxicity, neurotoxicity, and cardiotoxicity. ${ }^{15}$ Further, rapid plasma elimination of gambogic acid cannot be avoided by these formulations. ${ }^{12,16}$ These defects greatly limit the therapeutic effect of gambogic acid. Therefore, it is necessary to develop structural modifications or a new dosage form of gambogic acid.

Conjugation of hydrophilic polymers with small molecule drugs to produce polymer-drug conjugate systems has been demonstrated to be a viable formulation strategy for utilizing hydrophobic drugs in a water-soluble manner, which may offer advantages over the corresponding parent drugs, including fewer side effects, improved solubility, passive tumor targeting, an improved pharmacokinetic profile, and 
lower plasma concentrations. This passive tumor targeting is achieved primarily through the enhanced permeability and retention effect. In past decades, conjugation of hydrophilic polymers with hydrophobic anticancer drugs (such as paclitaxel, doxorubicin, camptothecin, and curcumin) to produce polymer-drug conjugate systems has been proven to be a viable formulation strategy. ${ }^{17-25}$ A large number of hydrophilic polymers could be chosen as water-soluble macromolecule carriers; among them, poly(ethylene glycol) (PEG) is considered to be one of the best candidates. Several chemotherapeutic agents have been conjugated to PEG with covalent bonds, including a PEG-curcumin conjugate, a PEGpaclitaxel conjugate, and a PEG-camptothecin conjugate, resulting in homogeneous water-soluble prodrugs with an extended circulatory life and altered biodistribution. ${ }^{26-29}$ Tang et $\mathrm{al}^{30}$ reported that using PEG as a water-solubilizing unit was a useful strategy for increasing the water solubility of gambogic acid, which is hydrophobic. Ding et al $^{31}$ synthesized a series of gambogic acid-PEG conjugates with different amino acid and dipeptide spacers, which showed satisfactory water solubility compared with gambogic acid, and the circulatory retention time, biodistribution, and bioavailability of the conjugates were remarkably improved. However, the bioactivity and toxicity of gambogic acid-PEG conjugates in vivo have not been reported thus far.

On the basis of these studies, we developed a nanosized drug delivery system with the aims of increasing the aqueous solubility, improving the therapeutic efficacy, and reducing the toxicity of gambogic acid to normal tissues. In this study, an amphiphilic polymer-drug conjugate was prepared by condensation of low molecular weight monomethoxypoly(ethylene glycol) (mPEG)-2000 with gambogic acid $\left(\mathrm{GA}-\mathrm{mPEG}_{2000}\right)$ through an ester linkage, which then formed micelles. The physicochemical properties of the formulations were evaluated, and their in vitro cytotoxicity, in vivo antitumor effect, and toxicity were also investigated.

\section{Materials and methods Materials}

Gamboge resin was obtained from Chengdu Herb Market (Sichuan, People's Republic of China). mPEG (average molecular weight 2,000) and Dulbecco's Modified Eagle's Medium were purchased from Sigma-Aldrich. Gambogic acid was prepared and purified in our laboratory. Acetonitrile of high-performance liquid chromatography (HPLC) grade was purchased from Fisher Scientific (Loughborough, UK). Chloroform, methanol (analytical grade), 1-ethyl-3-(3-dimethylaminopropyl)-carbodiimide hydrochloride (EDC), and N-hydroxysuccinimide were purchased from Chengdu Kelong Chemical Co, Ltd (Chengdu, People's Republic of China). Other reagents were of analytical grade. Deionized water ( $>18$ MU, Purelab ${ }^{\circledR}$; Classic Components Corporation, Torrance, CA, USA) was used in all experiments.

\section{Cells and animals}

Murine colon adenocarcinoma cells (C26) and murine melanoma cells (B16-F10) were purchased from the American Type Culture Collection (Rockville, MD, USA). The cells were grown in Dulbecco's Modified Eagle's Medium supplemented with 10\% fetal bovine serum (Sigma-Aldrich). Primary human umbilical vein endothelial cells (HUVECs) were isolated from human umbilical cord veins and grown in EBM-2 (endothelial basal medium-2) with Single Quots (Lonza, Walkersville, MD, USA) containing vascular endothelial growth factor and other growth factors. HUVECs at passages 2-4 were used for all experiments. The above cells were maintained at $37^{\circ} \mathrm{C}$ in a humidified incubator containing $5 \% \mathrm{CO}_{2}$.

$\mathrm{Balb} / \mathrm{c}$ and $\mathrm{C} 57 \mathrm{BL} / 6 \mathrm{~J}$ mice (age 6-8 weeks, weight 18-22 g) were used for the in vivo antitumor tests and toxicity studies. The animals were purchased from the Laboratory Animal Center of Sichuan University, and housed in sexsegregated cages at a controlled temperature of $20^{\circ} \mathrm{C}-22^{\circ} \mathrm{C}$, a relative humidity of $50 \%-60 \%$, and 12 -hour light-dark cycles. The animals were provided with standard laboratory chow and tap water ad libitum. After a 1-week acclimation period, the animals were used for the experiments. All animal procedures were performed following the protocol approved by the Institutional Animal Care and Treatment Committee of Sichuan University (Chengdu, People's Republic of China). All mice were treated humanely throughout the experimental period.

\section{Measurements}

${ }^{1} \mathrm{H}$ nuclear magnetic resonance (NMR) spectra were determined on an Avance 400 (Bruker Corporation, Ettlingen, Germany) spectrometer $(400 \mathrm{mHz})$ using deuterated chloroform as the solvent. Ultraviolet-visible spectra were recorded on a UV-2401 PC spectrophotometer (Shimadzu, Tokyo, Japan). HPLC detection was done on a 2996 detector (Waters Corporation, Milford, MA, USA). Chromatographic separations were performed on a reversed phase $\mathrm{C}_{18}$ column (4.6 mm $\times 150 \mathrm{~mm}, 5 \mu \mathrm{m}$, SunFire; Waters Corporation). The column temperature was kept at $28^{\circ} \mathrm{C}$, and the detection wavelength was $292 \mathrm{~nm}$. Acetonitrile/0.1\% formic acid $(80 / 20, \mathrm{v} / \mathrm{v})$ solution was used as the mobile phase, and the flow rate was $1 \mathrm{~mL}$ per minute. 


\section{General synthesis method for GA-mPEG ${ }_{2000}$ conjugates}

Isolation and purification of gambogic acid

Fifty grams of dry gamboge resin was suspended in $150 \mathrm{~mL}$ of pyridine and stirred at $80^{\circ} \mathrm{C}-90^{\circ} \mathrm{C}$ for 30 minutes to form a pyridine salt of gambogic acid. After filtering through Kieselguhr (Sigma-Aldrich), $20 \mathrm{~mL}$ of water was added to the filtrate. The mixture was cooled to $4^{\circ} \mathrm{C}$ overnight, and the precipitate was collected and washed with pyridine solution $(70 \%, v / v)$ and water several times. After drying under vacuum, the resulting yellow powder was dissolved in $250 \mathrm{~mL}$ of ethyl ether and heated under reflux for 30 minutes, then filtered, concentrated, and precipitated using petroleum ether; the yellow precipitate of the pyridine salt of gambogic acid was then collected and dried. The solid obtained was dissolved in $120 \mathrm{~mL}$ of ethyl ether and washed with aqueous $\mathrm{HCl}(1 \mathrm{M})$ and water. The ether solution was then dried over sodium sulfate and evaporated to yield an orange powder of $3.8 \mathrm{~g}$ gambogic acid (purity $98.6 \%$, w/w, analyzed by HPLC).

\section{Synthesis of GA-mPEG 2000}

Gambogic acid (345 mg, $0.55 \mathrm{mmol})$, EDC (191.7 mg, $1 \mathrm{mmol}$ ), and 4-dimethylaminopyridine (61 $\mathrm{mg}, 0.5 \mathrm{mmol})$ were dissolved in $10 \mathrm{~mL}$ of $\mathrm{CH}_{2} \mathrm{Cl}_{2}$ in an ice water bath, and $\mathrm{mPEG}_{2000}(1 \mathrm{~g}, 0.5 \mathrm{mmol})$ was then added. The resulting mixture was stirred overnight at room temperature. The solution was washed in $1 \mathrm{M} \mathrm{HCl}(3 \times 10 \mathrm{~mL})$, water $(2 \times 10 \mathrm{~mL})$, and brine $(2 \times 10 \mathrm{~mL})$, dried over $\mathrm{Mg}_{2} \mathrm{SO}_{4}$, and concentrated in vacuo. The precipitate was suspended in $10 \mathrm{~mL}$ of ethyl ether under stirring for 30 minutes to remove the free gambogic acid. The solution was filtered and the yellow precipitate was obtained. The crude product was further purified by silica gel column chromatography using a step gradient of methanol $(2 \%-5 \%)$ in $\mathrm{CH}_{2} \mathrm{Cl}_{2}$ to remove the free $\mathrm{mPEG}_{2000}$, giving rise to GA-mPEG ${ }_{2000}(747 \mathrm{mg}, 57 \%)$ as a buff-colored solid. A single spot was visualized with iodine vapor by thin liquid chromatography analysis (dichloromethane to methanol, 10:1). The ${ }^{1} \mathrm{H}$ NMR spectrum of GA-mPEG ${ }_{2000}$ was studied using an Avance 400 spectrometer $(400 \mathrm{mHz})$, and $\mathrm{CH}_{3} \mathrm{Cl}$ was used as the solvent.

\section{Preparation and characterization of GA-mPEG ${ }_{2000}$ micelles}

GA-mPEG ${ }_{2000}$ micelles were prepared using a direct dissolution method assisted by ultrasonication. GA-mPEG ${ }_{2000}$ $(84 \mathrm{mg}$ ) was dissolved in $10 \mathrm{~mL}$ of distilled water and sonicated at $25^{\circ} \mathrm{C}$ for 1 minute. The control sample was prepared by dissolving $22 \mathrm{mg}$ of L-arginine solubilizer and
$20 \mathrm{mg}$ of gambogic acid (GA-L) in $10 \mathrm{~mL}$ of distilled water and then sonicating the sample at $25^{\circ} \mathrm{C}$ for 3 minutes. The final concentrations of GA-mPEG ${ }_{2000}$ in the micelles and gambogic acid in the GA-L solution were determined by HPLC assay. A direct observation method was used to evaluate the solubility of the GA-mPEG ${ }_{2000}$ conjugates, and the equivalent solubility value was calculated depending on the measured solubility.

The particle size and zeta potential of the micelles were determined by dynamic light scattering using a Zetasizer Nano ZS-90 instrument (Malvern Instruments, Malvern, UK). The sample suspension (gambogic acid concentration $2 \mathrm{mg} / \mathrm{mL}$ ) was diluted ten times with deionized water before measurement. The refractive index was 1.330 . The temperature was kept at $25^{\circ} \mathrm{C}$ during the measuring process. All tests were run in triplicate, and mean values are reported.

The morphology of the prepared micelles was observed by transmission electron microscopy (TEM) and atomic force microscopy (AFM). For TEM, the samples were diluted with distilled water and placed on a copper grid covered with nitrocellulose. The samples were negatively stained with phosphotungstic acid and dried at room temperature, after which images were taken using a TEM device (H-6009IV; Hitachi, Japan). For AFM, the prepared micelle suspension was diluted with deionized water and deposited onto freshly cleaved mica lamella. The sample was dried for 3 hours at room temperature. AFM images were taken by tapping mode in air on an AFM device (SPA400; Seiko, Tokyo, Japan).

After preparation, the micelles were kept at $4^{\circ} \mathrm{C}$ and $25^{\circ} \mathrm{C}$, respectively, and the stability of the formulations was evaluated qualitatively by observation of aggregates and particle size measurement. A homogeneous solution implies stability of the drug system, whereas the presence of precipitation or marked changes of particle size indicates instability.

\section{Molecular modeling study Construction of three-dimensional GA-mPEG 2000 structures}

First, gambogic acid was built with MarvinSketch (http:// www.chemaxon.com) and optimized at the molecular mechanical level using the MMFF94 method. ${ }^{32}$ Meanwhile, $\mathrm{mPEG}_{2000}$ was constructed using Hyperchem ${ }^{33}$ software (Hypercube Inc., Gainesville, FL, USA). The gambogic acid and $\mathrm{mPEG}_{2000}$ structures were then merged together in the Hyperchem workspace (Figure 1A). GA-mPEG ${ }_{2000}$ was then optimized at the molecular mechanical level using the orthogonal partial least squares ${ }^{34}$ method with the steepest descent algorithm. It was further optimized at the same 


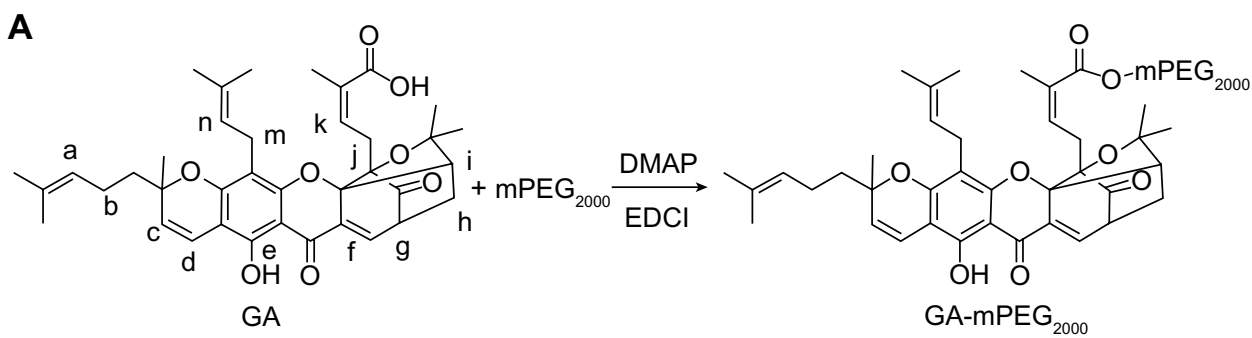

B

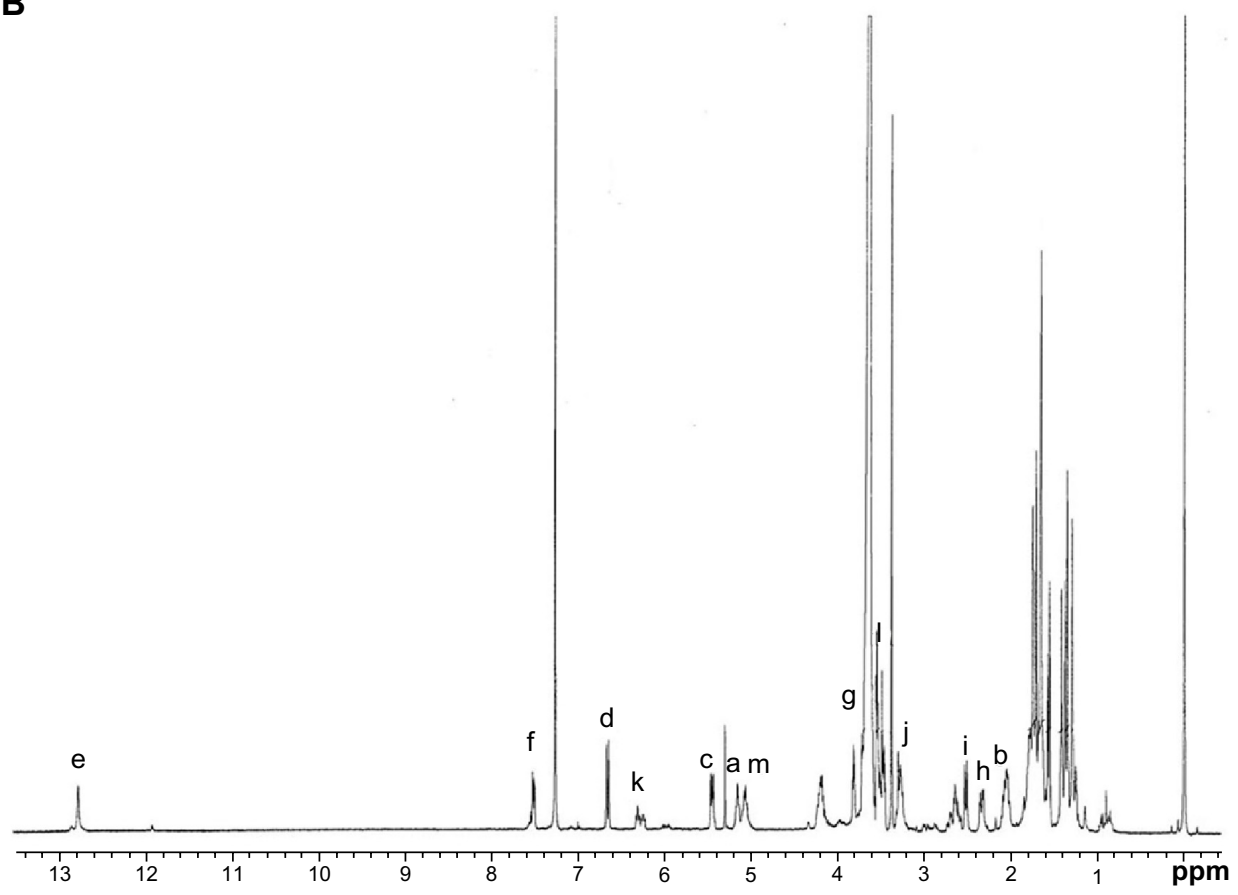

Figure I Synthesis and identification of $\mathrm{GA}-\mathrm{mPEG}_{2000}$ copolymers. (A) Chemical reaction scheme for preparing the $\mathrm{GA}_{-} \mathrm{mPEG}_{2000}$ conjugate. (B) 'H nuclear magnetic resonance spectrum of $\mathrm{GA}-\mathrm{mPEG}_{2000}$ in $\mathrm{CD}_{3} \mathrm{Cl}$.

Abbreviations: GA, gambogic acid; $\mathrm{mPEG}_{2000}$, methoxy poly(ethylene glycol) methyl ether (molecular weight 2,000); DMAP, 4-dimethylaminopyridine; EDC, I-ethyl-3-(3dimethylaminopropyl)-carbodiimide hydrochloride.

level with the molecular mechanical plus method ${ }^{35}$ using the Fletcher-Reeves ${ }^{36}$ algorithm.

Next, the optimized GA-mPEG ${ }_{2000}$ was subjected to a series of molecular dynamics simulations so as to obtain a lower energy minimum. The solvent effect was implicitly considered using the CHARMM27 $7^{37,38}$ force field in the process of molecular dynamics simulation. The molecular dynamics simulations included three stages: heating from $0 \mathrm{~K}$ to $400 \mathrm{~K}$, simulating at $400 \mathrm{~K}$, cooling from $400 \mathrm{~K}$ to $300 \mathrm{~K}$, and running at $300 \mathrm{~K}$. At each stage, the simulated time is set to 100 picoseconds.

\section{Simulation on self-assembly of GA-mPEG ${ }_{2000}$}

Two GA-mPEG ${ }_{2000}$ molecules, the structures of which had been annealed using molecular dynamics, were first merged together. Two kinds of conformations were purposefully arranged. In the first conformation, the gambogic acid moieties of the two molecules were arranged face-to-face. In the second conformation, they were arranged back-to-back. They were then simulated using Langevin dynamics. The friction coefficient and random seed were set as 0.05 per picosecond and $-1,111$, respectively. The solvation effect was treated as mentioned above. Simulation times for the first conformation and the second conformation were set to 100 picoseconds and 300 picoseconds, respectively.

\section{In vitro drug release}

Release of gambogic acid from the GA-L formulation and GA-mPEG ${ }_{2000}$ micelles in vitro was monitored by a dialysis method. Dialysis was carried out at $37^{\circ} \mathrm{C}$ using Spectra/Por dialysis membranes (Spectrum Laboratories, Inc, Rancho Dominguez, CA, USA) with a molecular weight cutoff of $1 \mathrm{kDa}$ and phosphate-buffered saline $(\mathrm{pH}$ 7.4) as the sink solution. The molecular weight cutoff of the dialysis membrane only allows for diffusion of the free drug. The initial concentration of gambogic acid in the GA-L formulations 
was $20 \mathrm{mg} / \mathrm{mL}$ and the initial concentration of GA-mPEG ${ }_{2000}$ in the micelles was $84 \mathrm{mg} / \mathrm{mL}$ (gambogic acid equivalent concentration, $20 \mathrm{mg} / \mathrm{mL}$ ). At scheduled intervals, $1 \mathrm{~mL}$ of the dialysis medium was collected and the same volume of fresh medium was added immediately. The concentration of gambogic acid in the dialysis medium was monitored by HPLC assay. The percentage release was calculated according to the following equation:

Drug release percentage $(\%)=\left(\mathrm{W}_{\text {release }} / \mathrm{W}_{\text {total }}\right) \times 100 \%$

where $\mathrm{W}_{\text {total }}$ is the total amount of gambogic acid in the GA-L formulation or micelles and $\mathrm{W}_{\text {release }}$ is the amount of gambogic acid released from the GA-L formulation or GA-mPEG ${ }_{2000}$ micelles into the dialysis medium.

\section{Cytotoxicity assay}

The in vitro cytotoxicity of the conjugates was quantified by measuring the $\mathrm{IC}_{50}$ (drug concentration inhibiting $50 \%$ of cells) in a murine melanoma cell line (B16-F10), a colon carcinoma cell line (C26), and HUVECs. B16-F10 and C26 cells were cultured in Dulbecco's Modified Eagle's Medium containing $10 \%$ heat-inactivated fetal calf serum at a density of $1 \times 10^{5}$ cells per $180 \mu \mathrm{L}$ per well in 96-well microtiter plates and allowed to proliferate at $37^{\circ} \mathrm{C}$ in a humidified incubator with $5 \% \mathrm{CO}_{2}$ for 24 hours. HUVEC cells were cultured in Dulbecco's Modified Eagle's Medium containing low serum growth supplement, 100 $\mathrm{IU} / \mathrm{mL}$ penicillin, and $100 \mu \mathrm{g} / \mathrm{mL}$ streptomycin at a density of $1 \times 10^{5}$ cells per $180 \mu \mathrm{L}$ per well in 96-well microtiter plates and allowed to proliferate at $37^{\circ} \mathrm{C}$ in a humidified incubator with $5 \% \mathrm{CO}_{2}$ for 24 hours. Next, $20 \mu \mathrm{L}$ serial dilutions of gambogic acid and GA-mPEG ${ }_{2000}$ (final equivalent gambogic acid concentration, $0.1-3.2 \mathrm{mg} / \mathrm{mL}$ ) were added to the respective wells, and all samples were prepared and measured in quintuplicate for each concentration. After 48 hours of incubation, $20 \mu \mathrm{L}$ of 3-(4,5-dimethylthiazol-2-yl)-2,5 diphenyl tetrazolium bromide (MTT, $5 \mathrm{mg} / \mathrm{mL}$ per well) was added and the plates were incubated for a further 4 hours. The supernatant was then removed, followed by addition of $150 \mu \mathrm{L}$ of dimethylsulfoxide to each well. Fifteen minutes later, the absorbance at $570 \mathrm{~nm}$ was detected using a microplate reader and the $\mathrm{IC}_{50}$ values for each compound were calculated from absorbance versus dilution factor plots.

\section{In vivo tumor model and treatment plan}

The antitumor activity of GA-mPEG ${ }_{2000}$ micelles was investigated in the B16-F10 and C26 models. In the B16-F10 model, $\mathrm{C} 57 \mathrm{BL} / 6 \mathrm{~J}$ mice were injected subcutaneously with $50 \mu \mathrm{L}$ of cell suspension containing $5 \times 10^{5}$ B16-F10 cells on day 0 . In the $\mathrm{C} 26$ model, Balb/c mice were injected subcutaneously into the right flank with $50 \mu \mathrm{L}$ of $\mathrm{C} 26$ cell suspension containing $2 \times 10^{6}$ cells at day 0 . Once tumors reached $150-250 \mathrm{~mm}^{3}$ in volume, the mice were randomized into three groups (ten mice per group). Tumor-bearing mice were injected intravenously every 2 days for 2 weeks with $100 \mathrm{~mL}$ of normal saline (control), GA-L (4 mg/kg bodyweight), or GA-mPEG ${ }_{2000}$ micelles ( $16.8 \mathrm{mg} / \mathrm{kg}$ body weight, gambogic acid $4 \mathrm{mg} / \mathrm{kg}$ equivalent), respectively. Tumor measurements were performed (according to the formula $0.52 \times$ length $\times$ width $^{2}$ ) and the mice were weighed and examined for antitumor activity every two (B16F10 model) or three (C26 model) days. Mouse survival times were observed to document further the antitumor activity and toxicity of GA-L and GA-mPEG ${ }_{2000}$ micelles.

\section{Statistical analysis}

The data are expressed as the mean and standard deviation. Statistical differences were determined using the Student's $t$-test. Differences were considered to be statistically significant at $P<0.05$.

\section{Results}

\section{Synthesis and identification of GA-mPEG 2000}

The synthesis of GA-mPEG ${ }_{2000}$ was according to the references in the literature with some modifications. ${ }^{30,39}$ Hydroxyterminated $\mathrm{mPEG}_{2000}$ was directly attached to gambogic acid at the 30-carboxy group. The main advantage of coupling one gambogic acid molecule at one end of $\mathrm{mPEG}_{2000}$ is the high selectivity of the only one hydroxyl in $\mathrm{mPEG}_{2000}$, which results in high yield and purity of GA-mPEG ${ }_{2000}$. The $\mathrm{mPEG}_{2000}$ was reacted with excess gambogic acid to guarantee that $\mathrm{mPEG}_{2000}$ was totally reacted. However, there was still some free $\mathrm{mPEG}_{2000}$ observed with iodine vapor by thin layer chromatography (dichloromethane to methanol, 10:1). The crude product was firstly purified in ethyl ether to remove free gambogic acid and then purified by gradient elution column chromatography.

Figure 1A shows the synthesis procedure for GA$\mathrm{mPEG}_{2000}$. The ${ }^{1} \mathrm{H}$ NMR spectrum of GA-mPEG ${ }_{2000}$ demonstrates successful synthesis of GA-mPEG ${ }_{2000}$. Figure 1B shows a multiplet at $\delta 3.58-3.72(\mathrm{ppm})$ that was attributed to the repeating units in $\mathrm{mPEG}_{2000}$, and the peaks at 3.38 ppm were assigned to the three methyl protons in $\mathrm{mPEG}_{2000}$. Protons of gambogic acid can be found at $12.78 \mathrm{ppm}(6-\mathrm{H})$, $7.50 \mathrm{ppm}(10-\mathrm{H}), 6.65 \mathrm{ppm}(4-\mathrm{H}), 6.33-6.23 \mathrm{ppm}(27-\mathrm{H})$, 5.48-5.42 ppm (3-H), 5.16-5.07 ppm (32-H, 37-H), $3.82 \mathrm{ppm}$ 
(11-H), 3.58-3.54 ppm (31a-H), 3.50-3.46 ppm (31b-H), 3.30-3.26 ppm (26-H), 2.52 ppm (22-H), 2.32 ppm (21a-H), and $2.09-2.02 \mathrm{ppm}(36-\mathrm{H})$, and all of the other multiple signals at $1.78-1.30$ came from the protons in gambogic acid. These results demonstrate further that the conjugate was successfully prepared.

\section{Characterization of GA-mPEG 2000 micelles}

Solubility of GA-mPEG ${ }_{2000}$ conjugates

The appearance of the prepared GA-mPEG ${ }_{2000}$ micelles is shown in Figure 2. Gambogic acid formed a turbid yellow suspension in water, indicating that gambogic acid could not be dissolved in aqueous solution (Figure 2A), whereas a clear and transparent solution of GA-mPEG ${ }_{2000}$ micelles could be observed (Figure 2B), indicating its good solubility in water. Compared with the extremely poor solubility of gambogic acid $(0.5 \mu \mathrm{g} / \mathrm{mL}), \mathrm{GA}_{-} \mathrm{MPEG}_{2000}$ exhibited satisfactory aqueous solubility, being $2.7 \times 10^{5}$-fold $(135.8 \mathrm{mg} / \mathrm{mL})$ that of gambogic acid.

\section{Particle size and morphology of GA-mPEG ${ }_{2000}$ micelles}

The GA-mPEG ${ }_{2000}$ micelles were suitable for an injectable formulation with a small particle size. The mean $( \pm$ standard deviation) vesicle diameter of the micelles was $9.41 \pm 2.91 \mathrm{~nm}(\mathrm{n}=3)$ as determined by dynamic light scattering, and the polydispersity index (PDI) was $0.193 \pm 0.072$ (Figure 2B). Further, AFM and TEM analyses confirmed that the micelles were spheroids with a regular shape and a size
A

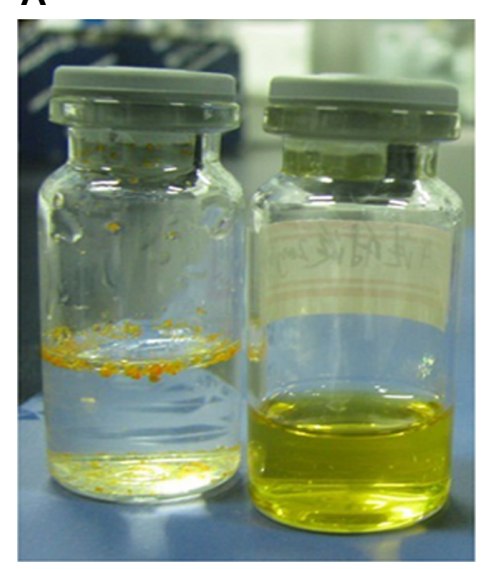

C

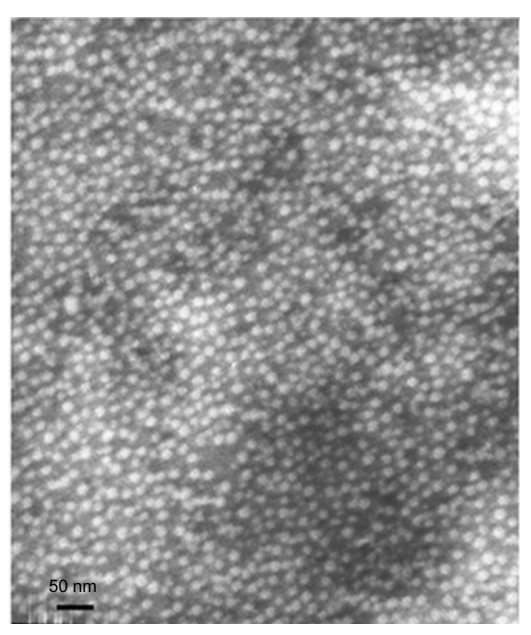

B

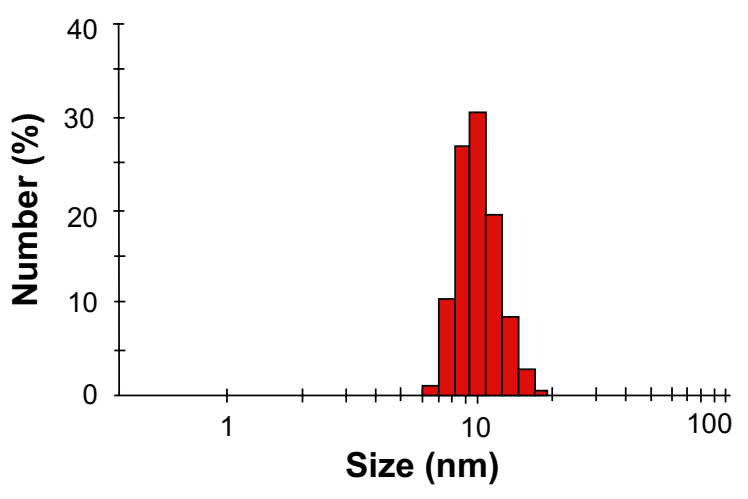

D

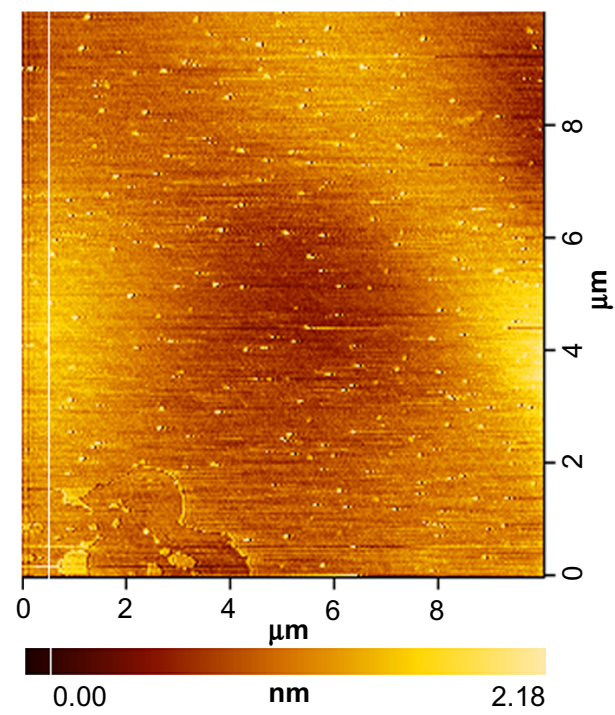

Figure 2 Preparation and characterization of GA-mPEG 2000 micelles. (A) Appearance of gambogic acid in various dosage forms (left) mixture of gambogic acid and water, (right) GA-mPEG 2000 micelles. (B) Size distribution of $\mathrm{GA}^{2000} \mathrm{mPEG}_{2000}$ micelles. (C) Transmission electron microscopic images of typical GA-mPEG 2000 micelles. (D) Atomic force microscopic images of typical GA-mPEG 2000 micelles.

Abbreviations: $\mathrm{GA}$, gambogic acid; $\mathrm{mPEG}_{2000}$, methoxy poly(ethylene glycol) methyl ether (molecular weight 2,000). 
distribution of $<50 \mathrm{~nm}$ (Figure 2C-D). The diameter of the micelles observed by AFM and TEM agreed with the results of the particle size analysis. These results demonstrate that the GA-mPEG ${ }_{2000}$ micelles were uniformly dispersed and had a very narrow particle size distribution.

\section{Stability of GA-L and GA-mPEG ${ }_{2000}$ micelles}

From observation of the aggregates, we found that the GA-L solution became turbid after 3 days and yellow precipitation could be observed after 5 days, indicating that the GA-L solution was not a stable formulation, whereas the GA$\mathrm{mPEG}_{2000}$ micelle solution remained clear and transparent for 30 days. From particle size measurement of the GA$\mathrm{mPEG}_{2000}$ micelles, we found that the average size and PDI of the micelles did not change significantly over 30 days (Figure 3). These results indicate that the GA-mPEG 2000 micelle formulation was a homogeneous and stable drug system.

\section{Molecular modeling study}

\section{Three-dimensional structure of GA-mPEG 2000}

As demonstrated in Figure 4A, the initial conformation of GA-mPEG ${ }_{2000}$ was a curve-like $\mathrm{mPEG}_{2000}$ covalently linking with gambogic acid. Kinetic simulations were done to find
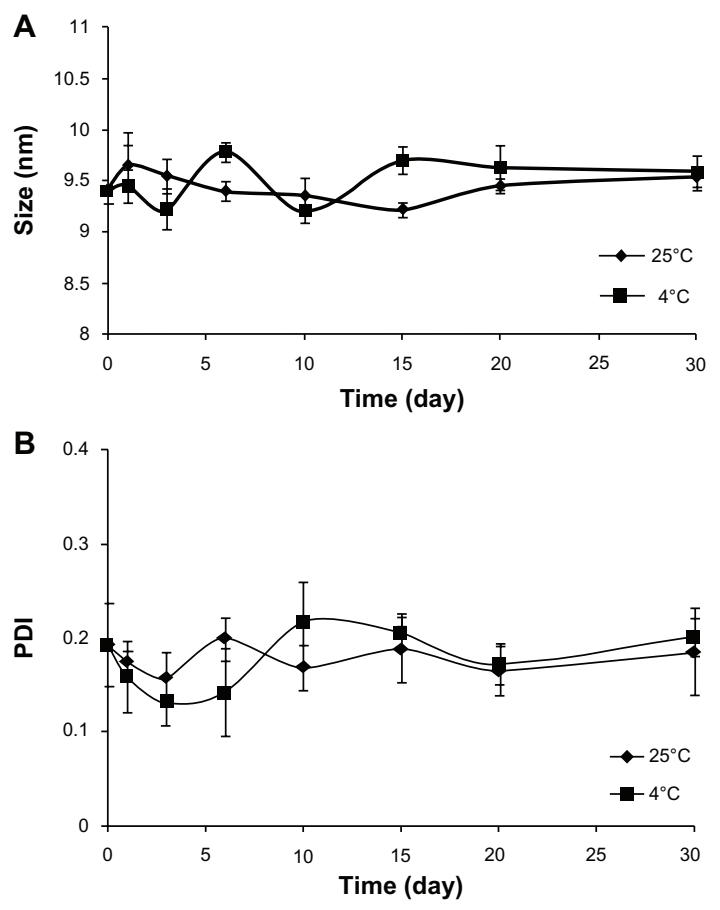

Figure 3 Stability of GA-mPEG ${ }_{2000}$ micelles. (A) Average size of GA-mPEG ${ }_{2000}$ micelles stored at $25^{\circ} \mathrm{C}$ and $4^{\circ} \mathrm{C}$. (B) PDI of GA-mPEG ${ }_{2000}$ micelles stored at $25^{\circ} \mathrm{C}$ and $4^{\circ} \mathrm{C}$.

Notes: $\mathrm{n}=3$ samples per group per time point; bars, mean \pm standard deviation. Abbreviations: GA, gambogic acid; $\mathrm{mPEG}_{2000}$, methoxy poly(ethylene glycol) methyl ether (molecular weight 2,000); PDI, polydispersity index.
A
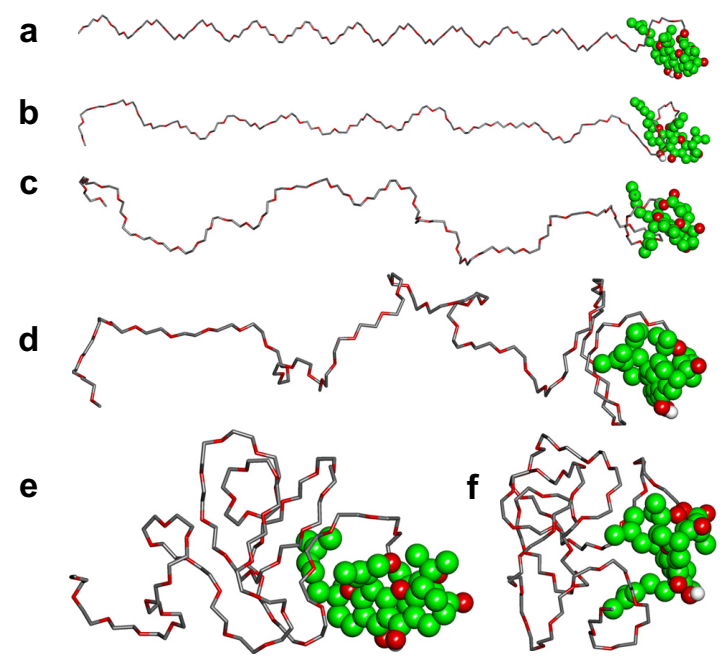

B
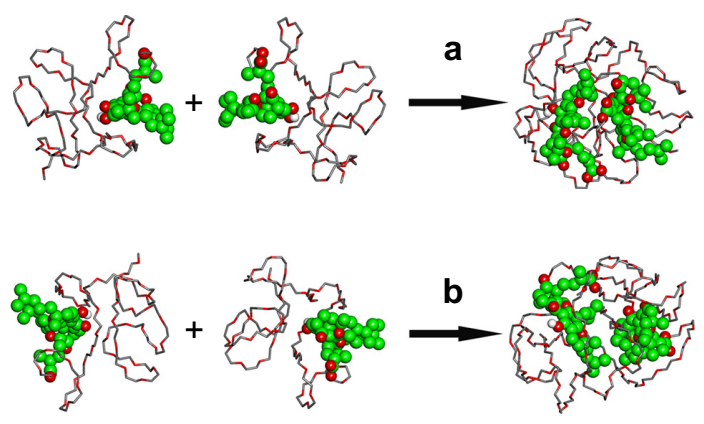

Figure 4 Molecular modeling studies of GA-mPEG 2000 micelles. (A) Conformations of GA-mPEG after being heated for 0 (a), 9.875 (b), 20.010 (c), 30.082 (d), 39.925 $(\mathbf{e})$, and 100 (f) picoseconds, respectively. (B) Interaction between GA-mPEG 2000 molecules resulting from face-to-face GA-GA initial conformation (a) and back-toback GA-GA conformation (b). GA was depicted with Corey-Pauling-Koltun space filling style, its carbon atoms were colored with green, and its oxygen atoms were colored with red.

Abbreviations: GA, gambogic acid; $\mathrm{mPEG}_{2000}$, methoxy poly(ethylene glycol) methyl ether (molecular weight 2,000).

an energy minimum for GA-mPEG ${ }_{2000}$. At the heating stage, GA-mPEG ${ }_{2000}$ was heated from $0 \mathrm{~K}$ to $400 \mathrm{~K}$ with a temperature step of $30 \mathrm{~K}$. It can be seen from Figure $4 \mathrm{~A}$ that the $\mathrm{mPEG}_{2000}$ moiety of GA-mPEG ${ }_{2000}$ changes its shape first and then gradually entwines around gambogic acid.

It must be a reasonable phenomenon due to the fact that the GA-mPEG ${ }_{2000}$ is in a polar solvent environment. As mentioned above, the solvation (water) effect was considered implicitly in the process of molecular dynamics simulation, because we know that gambogic acid is hydrophobic. The GA-mPEG ${ }_{2000}$ tries to increase its interaction with the polar solvent environment by reducing the hydrophobic moiety on its exposing surface. When $\mathrm{mPEG}_{2000}$ changes its shape and entwines around gambogic acid, the exposed hydrophobic area is reduced effectively. The equilibrated structure for GA-mPEG 2000 is shown in Figure 4A(f). 
Simulation on the self-assembly of GA-mPEG ${ }_{2000}$ In order to understand in detail the mechanism of interaction between GA-mPEG ${ }_{2000}$ molecules, Langevin dynamics simulations were conducted on clusters composed of two GA-mPEG ${ }_{2000}$ molecules. When two GA-mPEG ${ }_{2000}$ molecules were arranged so that the gambogic acid parts of the molecules were face-to-face (as shown in Figure 4B[a]), the gambogic acid moieties approached each other after 100 picoseconds of Langevin dynamics simulation. When two GA-mPEG ${ }_{2000}$ molecules were arranged so that the gambogic acid moieties were back-to-back (as shown in Figure 4B[b]), the gambogic acid parts of the two molecules were finally close to each other after 300 picoseconds of Langevin dynamics simulation. These results suggest that, in a polar solvent environment, the gambogic acid moieties of GA-mPEG ${ }_{2000}$ tend to move closer and form the core of the micelle, whereas the $\mathrm{mPEG}_{2000}$ moieties tend to stay in the external area and form the shell of the micelle, agreeing with the data in Figure 2.

\section{In vitro release profile}

A modified dialysis method was used to investigate the in vitro drug release behavior of the GA-L and GA-mPEG ${ }_{2000}$ micelles. The molecular weight cutoff of the dialysis membrane only allows for diffusion of free gambogic acid. As shown in Figure 5, the GA-mPEG ${ }_{2000}$ micelles showed a
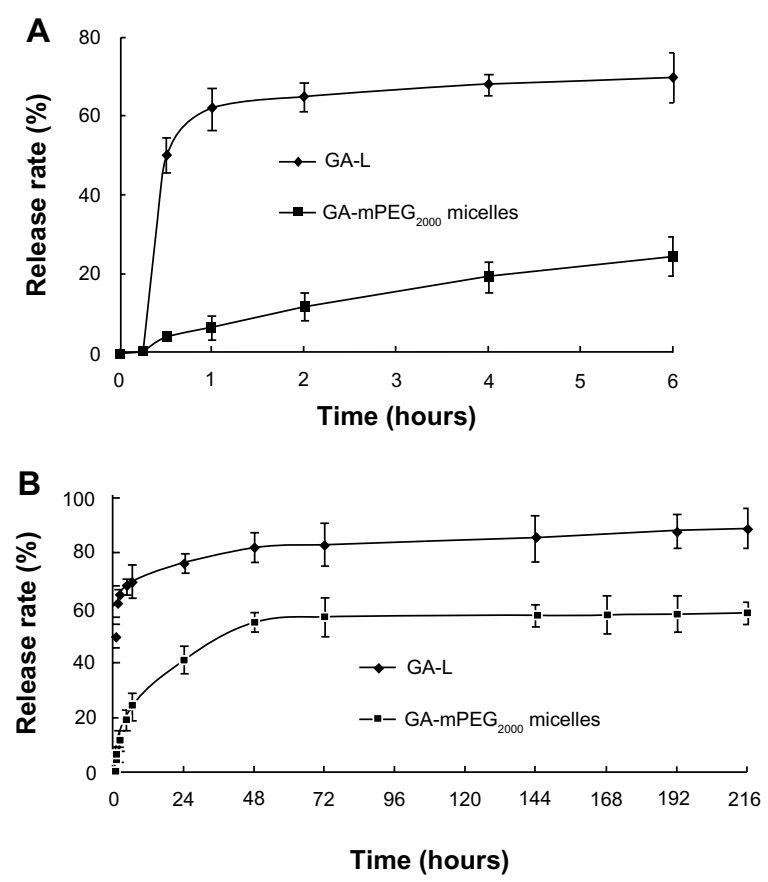

Figure 5 Time course of GA release from the GA-L formulation $(\downarrow)$ and GAmPEG $_{2000}$ micelles $(\mathbf{\square})$ at $37^{\circ} \mathrm{C}$ and $\mathrm{pH} 7.4$ in 6 hours (A) and 216 hours (B).

Notes: $n=3$ samples per group per time point; bars, mean \pm standard deviation. Abbreviations: GA, gambogic acid; $\mathrm{mPEG}_{2000}$, methoxy poly(ethylene glycol) methyl ether (molecular weight 2,000); L, L-arginine. much slower cumulative release rate compared with the rapid release profile for GA-L. Approximately $69 \%$ of the gambogic acid was released into the medium in the GA-L group within 6 hours, whereas only $24 \%$ of gambogic acid was released from the micelles. Over a 2 -week period, the mean cumulative release rate in the GA-mPEG ${ }_{2000}$ micelle group was much lower than that in the GA-L group $(58.30 \% \pm 4.28 \%$ versus $89.37 \% \pm 7.43 \%$, respectively). The in vitro drug release data show that gambogic acid could be released from the micelles in a sustained manner for an extended period.

\section{Cell cytotoxicity assay}

The in vitro biological efficacy of the GA-mPEG ${ }_{2000}$ micelles was evaluated against B16-F10, C26, and HUVEC cells using the MTT method. The data in Table 1 show that both GA-L and the micelles were cytotoxic to tumor cells and HUVEC cells. Compared with GA-L, the $\mathrm{IC}_{50}$ values for the GA- $\mathrm{mPEG}_{2000}$ micelles were 2.7-3.5 times greater than those for gambogic acid.

\section{Antitumor effects and toxicity of GA-L and $\mathrm{GA}^{-m P E \mathrm{PE}_{2000}}$ in vivo}

Mice subcutaneously injected with B16-F10 melanoma cells or $\mathrm{C} 26$ colon carcinoma cells were used to compare the antitumor activity of GA-L with that of GA-mPEG ${ }_{2000}$ micelles. In both models (Figures 6 and 7), tumor growth rates in mice treated with GA-L or GA- $\mathrm{mPEG}_{2000}$ micelles were markedly delayed, and GA-mPEG ${ }_{2000}$ micelles were more efficient than GA-L in suppressing growth of tumors. On day 20 after treatment, the $\mathrm{T} / \mathrm{C}$, defined as the ratio of the mean tumor volume in the treated group to that in the control group treated with normal saline, was $0.52(P<0.05)$ in mice treated with GA-L and $0.39(P<0.05)$ in mice treated with

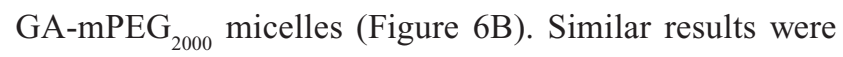
observed in $\mathrm{C} 26$ tumor-bearing mice (Figure 7B); on day 22 after treatment, the $\mathrm{T} / \mathrm{C}$ value was $0.35(P<0.05)$ in mice treated with GA-L and $0.20(P<0.01)$ in mice treated with GA-mPEG $_{2000}$ micelles. In addition, in both tumor models,

Table I Half maximal inhibitory concentration of different types of cells after incubation with the different drugs for 48 hours

\begin{tabular}{lll}
\hline & \multicolumn{1}{l}{ IC $_{50} \pm$ SD (GA equivalent, $\mu$ M) } \\
\cline { 2 - 3 } & GA & GA-mPEG ${ }_{2000}$ micelles \\
\hline BI6-FI0 & $0.33 \pm 0.05$ & $1.16 \pm 0.27$ \\
C26 & $0.80 \pm 0.16$ & $2.67 \pm 0.38$ \\
HUVEC & $0.81 \pm 0.09$ & $2.15 \pm 0.27$ \\
\hline
\end{tabular}

Abbreviations: $\mathrm{GA}$, gambogic acid; $\mathrm{IC}_{50}$, half maximal inhibitory concentration; $\mathrm{SD}$, standard deviation; HUVECs, human umbilical vein endothelial cells; $\mathrm{mPEG}_{2000}$, methoxy poly(ethylene glycol) methyl ether (molecular weight 2,000). 
A

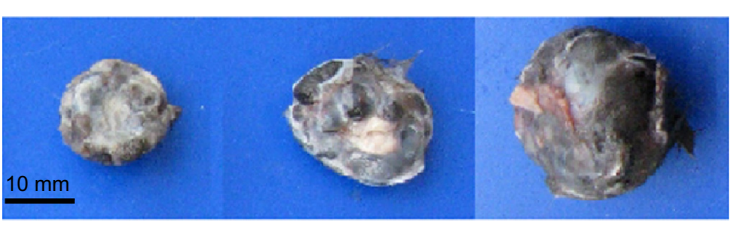

$\mathrm{GA}^{\mathrm{mPEG}}{ }_{2000}$

GA-L

C

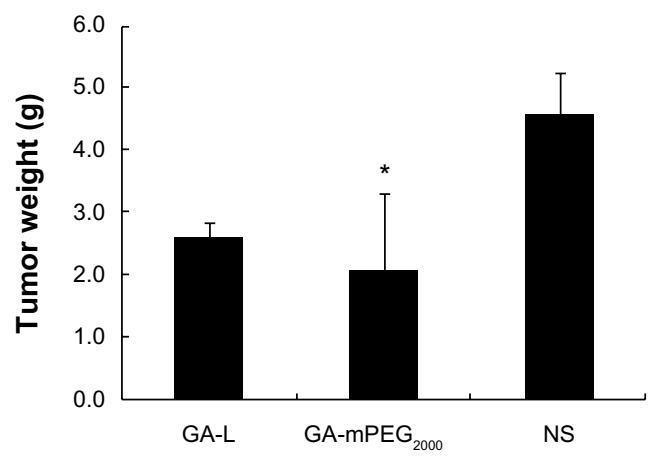

B

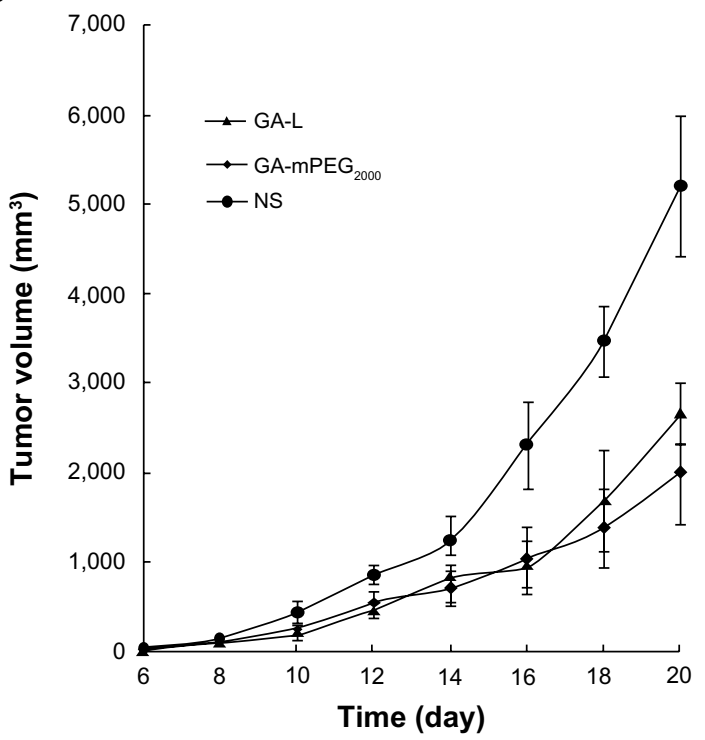

Figure 6 Antitumor study of GA and GA-mPEG ${ }_{2000}$ micelles in BI6-FI0 model. (A) Representative photographs of subcutaneous tumors in the GA-L group, GA-mPEG 2000

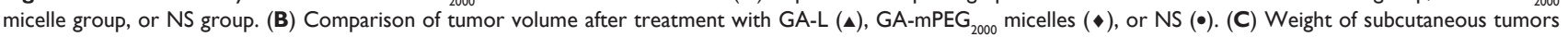
in each group.

Notes: $\mathrm{n}=5$ mice per group per time point; bars, mean \pm standard deviation. $* P<0.05$.

Abbreviations: GA, gambogic acid; $\mathrm{mPEG}_{2000}$, methoxy poly(ethylene glycol) methyl ether (molecular weight 2,000); L, L-arginine; NS, normal saline.

tumor weights in the GA-mPEG ${ }_{2000}$ micelle group after the final treatment were markedly lower than in the free drug and normal saline control group (Figures $6 \mathrm{C}$ and $7 \mathrm{C}$ ). In the B16F10 tumor-bearing mouse model (Figure 6C), mean tumor weights in the GA-mPEG ${ }_{2000}$ micelle group $(2.07 \pm 1.24 \mathrm{~g})$ decreased by $20 \%$ and $55 \%(P<0.05)$ compared with the GA-L group $(2.6 \pm 0.24 \mathrm{~g})$ and normal saline control group ( $4.51 \pm 0.65 \mathrm{~g}$ ), respectively. In the $\mathrm{C} 26$ tumor-bearing mouse model (Figure 7C), mean tumor weights in the GA-mPEG ${ }_{2000}$ micelle group $(0.504 \pm 0.42 \mathrm{~g})$ decreased by $58 \%$ and $83 \%$
A

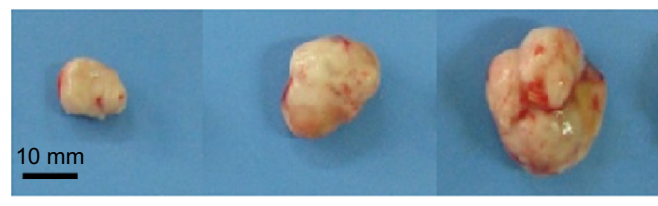

GA-mPEG ${ }_{2000}$

C

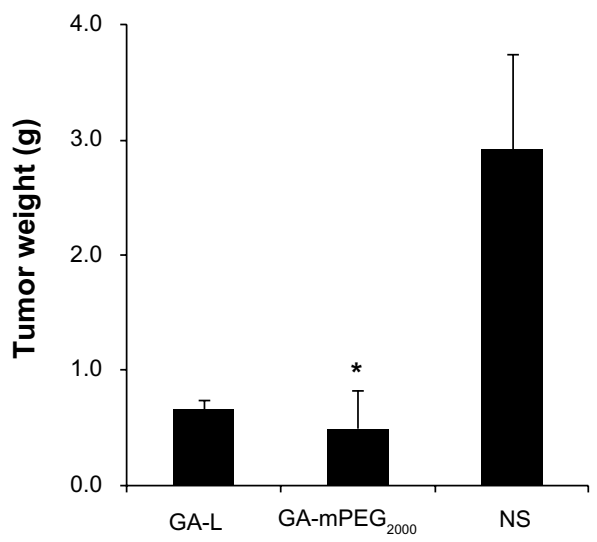

B

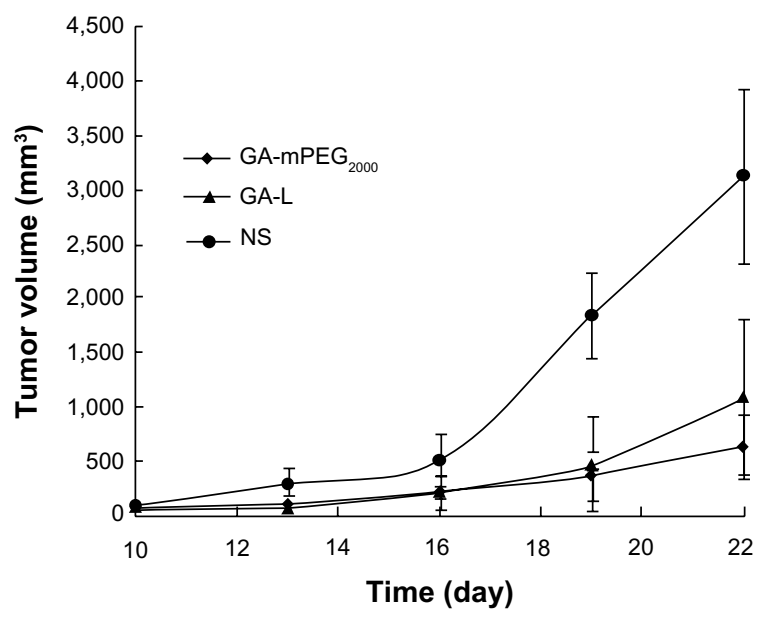

Figure 7 Antitumor study of GA and GA-mPEG ${ }_{2000}$ micelles in C26 model. (A) Representative photographs of subcutaneous tumors in the GA-L group, GA-mPEG 2000 micelle group, or NS group. (B) Comparison of tumor volume after treatment with GA-L $(\mathbf{\Delta}), \mathrm{GA}_{-\mathrm{mPEG}}{ }_{2000}$ micelles $(\bullet)$, or NS $(\bullet)$. (C) Weight of subcutaneous tumors in each group.

Notes: $n=5$ mice per group per time point; bars, mean \pm standard deviation. ${ }^{*} P<0.05$.

Abbreviations: $\mathrm{GA}$, gambogic acid; $\mathrm{mPEG}_{2000}$, methoxy poly(ethylene glycol) methyl ether (molecular weight 2,000); L, L-arginine; NS, normal saline. 
$(P<0.05)$ compared with the GA-L group $(1.2 \pm 0.72 \mathrm{~g})$ and normal saline control group $(2.922 \pm 0.83 \mathrm{~g})$, respectively. These results suggest that the GA-mPEG ${ }_{2000}$ micelles enhanced the antitumor effects of gambogic acid alone, and markedly delayed tumor growth rates in mice.

Throughout the experiment, the mice tolerated the scheduled doses of GA-mPEG ${ }_{2000}$ micelles and did not show obvious changes in fur, behavior, or eating. However, when mice were treated with GA-L intravenously, they struggled and demonstrated piloerection every time. As shown in Figure 8A and B, weight gain was normal in mice from the normal saline group, but decreased slightly in the GA-mPEG ${ }_{2000}$ micelle group, while that in the gambogic acid group was significantly lower than that of the other two groups. During our study, we did not observe any treatment-related mortality in the GA-mPEG ${ }_{2000}$ micelle group; however, mice in the GA-L group started to die after the third (B16-F10 model) and second (C26 model) drug treatments. Moreover, for both models, we observed a substantial increase in the life span of mice in the GA$\mathrm{mPEG}_{2000}$ micelle group. In the B16-F10 model, 50\% of mice treated with GA-mPEG ${ }_{2000}$ micelles survived for more than 24 days $(P<0.01$, Figure $8 \mathrm{C})$. In contrast, all mice treated with GA-L died within 18 days. In the $\mathrm{C} 26$ model, $70 \%$ of mice treated with $\mathrm{GA}-\mathrm{mPEG}_{2000}$ micelles survived for more than 31 days $(P<0.01$, Figure $8 \mathrm{D})$. In contrast, all mice treated with GA-L died within 28 days. These results suggest that GA-L is lethal in mice, whereas GA-mPEG ${ }_{2000}$ micelles decrease the toxicity of gambogic acid and prolong the lifespan of the animals.
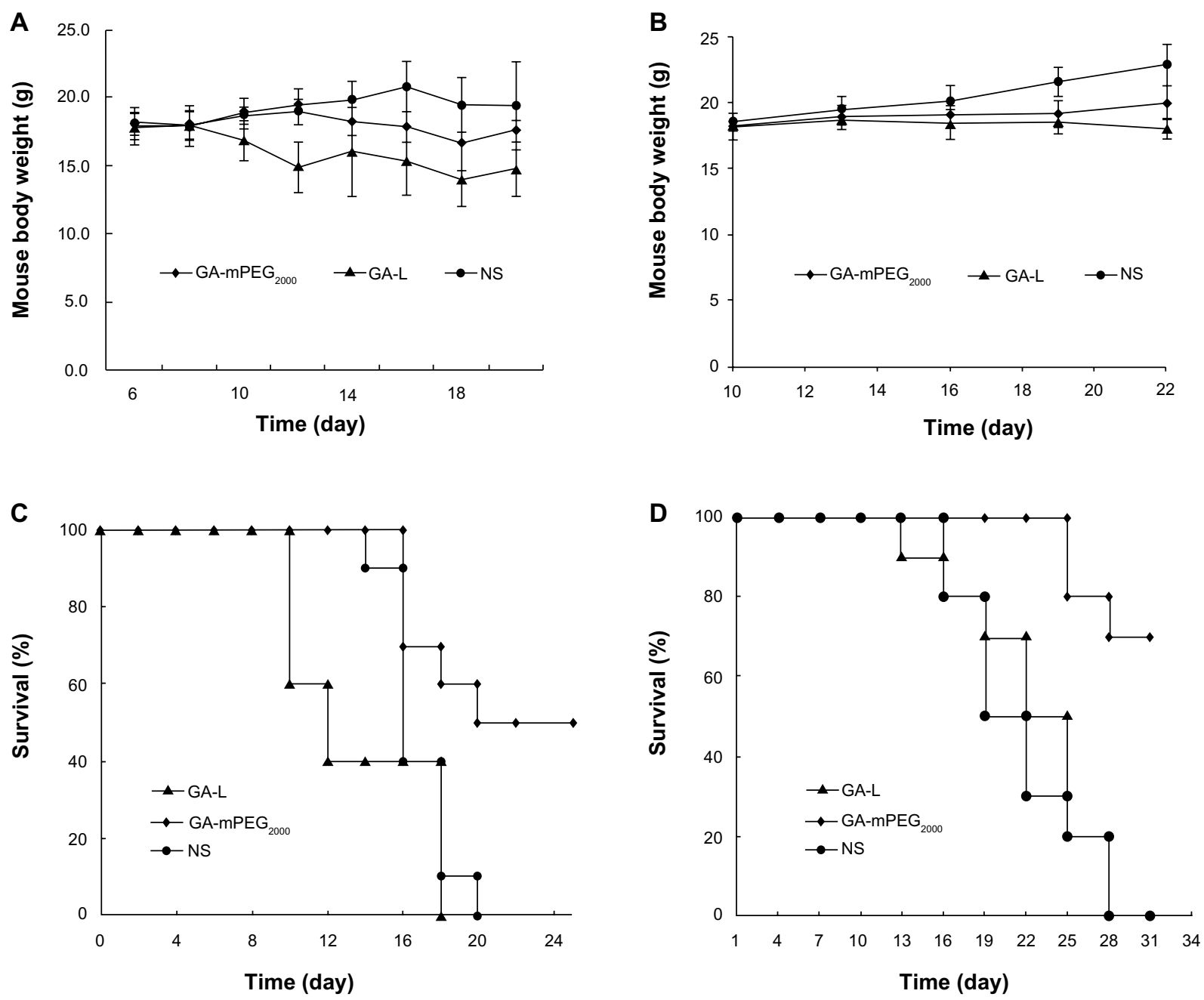

Figure 8 Toxicity study of GA and GA-mPEG 2000 micelles in BI6-FI0 and C26 models. (A) Curves for body weight in BI6-FI0 tumor-bearing mice after treatment with GA-L ( $\mathbf{\Delta})$, GA-mPEG 2000 micelles $(\bullet)$, or NS $(\bullet)$. (B) Curves for body weight in C26 tumor-bearing mice after treatment with GA-L $(\mathbf{\Delta})$, GA-mPEG ${ }_{2000}$ micelles $(\bullet)$, or NS $(\bullet)$.

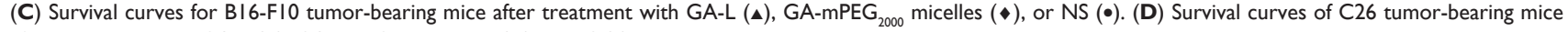
after treatment with GA-L $(\mathbf{\wedge}), \mathrm{GA}_{-\mathrm{mPEG}}{ }_{2000}$ micelles $(\bullet)$, or NS $(\bullet)$.

Notes: $\mathrm{n}=10$ mice per group per time point; bars, mean \pm standard deviation.

Abbreviations: GA, gambogic acid; $\mathrm{mPEG}_{2000}$, methoxy poly(ethylene glycol) methyl ether (molecular weight 2,000); L, L-arginine; NS, normal saline. 


\section{Discussion}

In this study, low molecular weight PEG (ie, $2 \mathrm{kDa}$ ) was conjugated to the hydrophobic drug, gambogic acid (molecular weight $628.75 \mathrm{Da}$ ), and this amphiphilic conjugate was used to form micelles as a colloidal carrier. As shown in Figure 2, the GA-mPEG ${ }_{2000}$ conjugate formed nanosized micelles with a mean particle size of about $10 \mathrm{~nm}$. These particles could be evenly dispersed in water, enabling the micelles to penetrate leaky vasculature and spontaneously accumulate in solid tumors via the enhanced permeability and retention effect. ${ }^{40}$ The maximum drug loading achieved in this formulation was $135.8 \mathrm{mg} / \mathrm{mL}$ of physically entrapped gambogic acid, indicating that the polymer-drug conjugate markedly improved the solubilization capacity of gambogic acid. Achievement of this high drug loading level highlights the influence of drug-excipient compatibility as demonstrated in previous studies. ${ }^{41}$

Over 30 days, the GA-mPEG ${ }_{2000}$ micelle solution remained clear and transparent, and the average size and PDI did not change significantly (Figure 3 ). Therefore, conjugating gambogic acid with biocompatible water-soluble $\mathrm{mPEG}_{2000}$ resulted in a homogeneous and stable dosage form in aqueous solution with high drug loading and a small particle size, which makes gambogic acid more suitable for intravenous administration.

From the in vitro drug release profile (Figure 5), we found that GA-mPEG ${ }_{2000}$ micelles had much slower release behavior compared with GA-L, and the gambogic acid in the GA-mPEG ${ }_{2000}$ micelles had greatly increased stability compared with GA-L. Drug release from micelles might be due to hydrolysis of the conjugate. ${ }^{42}$ This attenuated drug release suggests potential applicability of polymeric micelles as a controlled drug delivery system that could result in a more favorable pharmacokinetic profile in vivo and minimize exposure of healthy tissues, while increasing accumulation of drug at the tumor site..$^{43-45}$

An in vitro cytotoxicity study showed that GA- $\mathrm{mPEG}_{2000}$ micelles retain the cytotoxicity of gambogic acid to B16-F10, C26, and HUVECs, indicating that polymeric prodrugs do not lose the anticancer and antiangiogenetic activity of gambogic acid. However, compared with free gambogic acid, the $\mathrm{IC}_{50}$ values for the GA-mPEG ${ }_{2000}$ micelles were increased by 2.7-3.0-fold. This suggests that the cytotoxicity is mainly due to the native gambogic acid released from the GA-mPEG ${ }_{2000}$ micelles, and the relatively lower cytotoxicity seen might be induced by the slower release behavior of gambogic acid from micelles over 48 hours. These results are consistent with the drug release profile mentioned earlier.
Conjugation of drug molecules with biocompatible water-soluble polymers, eg, PEG, has been shown to increase their apparent aqueous solubility, provide protection from degradation, and/or improve their pharmacokinetics and biodistribution. ${ }^{46-49}$ Conjugation to PEG as a carrier could enable passive targeting to solid tumors via the enhanced permeability and retention effect..$^{50-52}$ Our in vivo antitumor study showed that GA-mPEG ${ }_{2000}$ micelles were more efficient in suppressing growth of tumors and prolonging the life span of mice compared with GA-L (Figures 6-8). The underlying mechanisms might involve three phases. First, aggregation of the GA-mPEG ${ }_{2000}$ conjugate to form micelles could protect the ester linkage from hydrolysis and protect the encapsulated drug from enzymatic degradation. Second, micelles with a small size could penetrate the leaky vasculature and accumulate in tumor tissue via the enhanced permeability and retention effect. Third, the micelles may release the drug slowly at tumor sites, resulting in higher drug concentrations in the tumor and lower drug concentrations in other tissues. Multiple mechanisms may be involved in the antitumor effect of gambogic acid, including induction of apoptosis, cell cycle arrest, telomerase inhibition, antiangiogenesis activity, and an antimetastasis effect. ${ }^{5-10}$ Our in vitro cytotoxicity study showed that gambogic acid kills tumor cells, and this also occurs in vivo. Gambogic acid can also inhibit angiogenesis, resulting in suppression of tumor growth in vivo. Therefore, the anticancer effect of GA-mPEG ${ }_{2000}$ micelles may be induced by inhibiting angiogenesis and directly killing tumor cells.

In this study, all mice showed abnormal changes in their fur and behavior during and after administration of GA-L, indicating that this formulation is irritating to mice. Interestingly, administration of the GA-mPEG ${ }_{2000}$ micelles did not induce such changes in these animals, suggesting that the nanosized assemblies of GA-mPEG ${ }_{2000}$ caused less toxicity and irritation compared with GA-L. Further, the body weight of mice in the GA-L group was significantly decreased after treatment, but not in the GA-mPEG ${ }_{2000}$ micelle group. Given that body weight loss is used as an indicator of the adverse effects of drugs and chemicals ${ }^{53}$ our results demonstrate the decreased toxicity of GA-mPEG ${ }_{2000}$ micelles compared with GA-L. Meanwhile, we observed high mortality in the GA-L group when tumor volume was $<300 \mathrm{~mm}^{3}$, suggesting that these deaths were not caused by the tumor but by drug toxicity. Encouragingly, we did not observe these phenomena in the GA- $\mathrm{MEG}_{2000}$ micelle group, suggesting that the GA-mPEG ${ }_{2000}$ micelles did not have lethal toxicity in mice. Our results further confirm the decreased toxicity of GA- 
$\mathrm{mPEG}_{2000}$ micelles compared with GA-L. Two underlying mechanisms might be involved. First, conjugation with $\mathrm{mPEG}_{2000}$ may decrease the toxicity of gambogic acid. ${ }^{31}$ Second, PEGylated micelles could prolong blood residence time and reduce the risk of nonspecific accumulation of the drug in the body. ${ }^{54}$ As a result, the drug might be more distributed at the tumor site, but less accumulated in normal tissue, thus reducing drug side effects.

To our knowledge, this work is the first concerning use of GA-mPEG ${ }_{2000}$ in melanoma and colon cancer therapy in vivo. Our results suggest that intravenous administration of GA-mPEG ${ }_{2000}$ micelles may have potential application in treating melanoma and colon cancer.

\section{Conclusion}

Biodegradable $\mathrm{mPEG}_{2000}$ conjugates of the natural antitumor agent gambogic acid were prepared. These GA-mPEG ${ }_{2000}$ conjugates could self-assemble into micelles with better solubility in water as compared with gambogic acid. Moreover, the GA-mPEG ${ }_{2000}$ micelles enhanced drug stability and antitumor effects, prolonged survival time, and were less toxic to mice than gambogic acid. Our results indicate that such polymer-drug conjugate systems could solve many of the problems associated with insoluble chemicals and natural anticancer agents, and at the same time achieve excellent properties in drug delivery systems.

\section{Acknowledgments}

This work was supported financially by the National Natural Science Foundation of China (81071251) and the Open Research Fund of State Key Laboratory Breeding Base of Systematic Research, Development and Utilization of Chinese Medicine. We acknowledge Shichao He and Wen Yang for their help with the synthesis and antitumor studies. We also acknowledge Joseph Rohr and Xianhuo Wang of the Department of Pathology and Microbiology at the University of Nebraska Medical Center, Omaha, NE, USA, for editing the manuscript.

\section{Disclosure}

The authors report no conflicts of interest in this work.

\section{References}

1. Auterhoff H, Frauendorf H, Liesenklas W, Schwandt C. [The chief constituents of gamboge resins. 1. Chemistry of gamboge]. Arch Pharm. 1962;295:833-846. German.

2. Ollis WD, Ramsay MVJ, Sutherland IO, Mongkolsuk S. The constitution of gambogic acid. Tetrahedron. 1965;21:1453-1470.
3. Cai SX, Jiang S, Zhang HZ, inventors. Derivatives of gambogic acid and analogs as activators of caspases and inducers of apoptosis. World Intellectual Property Organization Patent Application PCT/US2004/042292. December 17, 2004.

4. Zhao L, Guo QL, You QD, Wu ZQ, Gu HY. Gambogic acid induces apoptosis and regulates expressions of $\mathrm{Bax}$ and $\mathrm{Bcl}-2$ protein in human gastric carcinoma MGC-803 cells. Biol Pharm Bull. 2004;27: 998-1003.

5. Yu J, Guo QL, You QD, et al. Repression of telomerase reverse transcriptase mRNA and hTERT promoter by gambogic acid in human gastric carcinoma cells. Cancer Chemother Pharmacol. 2006;58: 434-443.

6. Guo QL, Lin SS, You QD, et al. Inhibition of human telomerase reverse transcriptase gene expression by gambogic acid in human hepatoma SMMC-7721 cells. Life Sci. 2006;78:1238-1245.

7. Liu W, Guo QL, You QD, Zhao L, Gu HY, Yuan ST. Anticancer effect and apoptosis induction of gambogic acid in human gastric cancer line BGC-823. World J Gastroenterol. 2005;11:3655-3659.

8. Qiang L, Yang Y, You QD, et al. Inhibition of glioblastoma growth and angiogenesis by gambogic acid: an in vitro and in vivo study. Biochem Pharmacol. 2008;75:1083-1092.

9. Yang Y, Yang L, You QD, et al. Differential apoptotic induction of gambogic acid, a novel anticancer natural product, on hepatoma cells and normal hepatocytes. Cancer Lett. 2007;256:259-266.

10. Sun S Y, Yue P, Kelloff GJ, et al. Identification of retinamides that are more potent than $\mathrm{N}$-(4-hydroxyphenyl) retinamide in inhibiting growth and inducing apoptosis of human head and neck and lung cancer cells. Cancer Epidemiol Biomarkers. 2001;10:595-601.

11. Liu YT, Hao K, Liu XQ, Wang GJ. Metabolism and metabolic inhibition of gambogic acid in rat liver microsomes. Acta Pharmacol Sin. 2006;27:1253-1258.

12. Hao K, Liu XQ, Wang GJ, Zhao XP. Pharmacokinetics, tissue distribution and excretion of gambogic acid in rats. Eur J Drug Metab Pharmacokinet. 2007;32:63-68.

13. Dai JG, inventor. The preparation of a kind of gambogic acid injection. CN Patent No 03131511.9, Dec 5, 2003.

14. You OD, Guo QL, Ke X, et al, inventors. The preparations of gambogic acid and its compound. CN Patent No 03132386.3, July 21, 2004.

15. Qi Q, You Q, Gu H, et al. Studies on the toxicity of gambogic acid in rats. J.Ethnopharmacol. 2008;117:433-438.

16. Han J, Xiao J, Wang H, et al. Measurement and correlation of taxol solubility in methanol, ethanol and methanol-water systems. J Chem Ind Eng. 2001;52:64-67.

17. Khandare J, Minko T. Polymer-drug conjugates: progress in polymeric prodrugs. Prog Polym Sci. 2006;31:359-397.

18. Satchi-Fainaro R, Duncan R, Barnes CM. Polymer Therapeutics for Cancer: Current Status and Future Challenges. Polymer Therapeutics II. Berlin, Germany: Springer; 2006.

19. Li H, Huo M, Zhou J, et al. Enhanced oral absorption of paclitaxel in N-deoxycholic acid-N, O-hydroxyethyl chitosan micellar system. J Pharm Sci. 2010;99:4543-4553.

20. Hou L, Fan Y, Yao J, et al. Low molecular weight heparin-all-transretinoid acid conjugate as a drug carrier for combination cancer chemotherapy of paclitaxel and all-trans-retinoid acid. Carbohydr Polym. 2011;86:1157-1166.

21. Kwon GS, Okano T. Polymeric micelles as new drug carriers. Adv Drug Deliv Rev. 1996;21:107-116.

22. Greenwald RB, Pendri A, Conover C, Gilbert C, Yang R, Xia J. Drug delivery systems. 2. Camptothecin 20-O-poly (ethylene glycol) ester transport forms. J Med Chem. 1996;39:1938-1940.

23. Sun M, Su X, Ding B, et al. Advances in nanotechnology-based delivery systems for curcumin. Nanomedicine. 2012;7:1085-1100.

24. Tang H, Murphy CJ, Zhang B, et al. Curcumin polymers as anticancer conjugates. Biomaterials. 2010;31:7139-7149.

25. Manju S, Sreenivasan K. Conjugation of curcumin onto hyaluronic acid enhances its aqueous solubility and stability. $J$ Colloid Interface Sci. 2011;359:318-325. 
26. Murphy CJ, Tang H, Van Kirk EA, Shen Y, Murdoch WJ. Reproductive effects of a pegylated curcumin. Reprod Toxicol. 2012;34:120-124.

27. Li J, Wang Y, Yang C, et al. Polyethylene glycosylated curcumin conjugate inhibits pancreatic cancer cell growth through inactivation of Jab1. Mol Pharmacol. 2009;76:81-90.

28. Ceruti M, Crosasso P, Brusa P, Arpicco S, Dosio F, Cattel L. Preparation, characterization, cytotoxicity and pharmacokinetics of liposomes containing water-soluble prodrugs of paclitaxel. $J$ Control Release. 2000;63:141-153.

29. Fleming AB, Haverstick K, Saltzman WM. In vitro cytotoxicity and in vivo distribution after direct delivery of PEG-camptothecin conjugates to the rat brain. Bioconjug Chem. 2004;15:1364-1375.

30. Tang X, Zhang P, Ye H, Zhang C, Shen W, Ping Q. Water-soluble gambogic acid PEGylated prodrugs: synthesis, characterization, physicochemical properties and in vitro hydrolysis. Pharmazie. 2008;63: 711-717.

31. Ding Y, Zhang P, Tang XY. PEG prodrug of gambogic acid: amino acid and dipeptide spacer effects. Polymer. 2012;53:1694-1702.

32. Halgren TA. Merck molecular force field. I. Basis, form, scope, parameterization, and performance of MMFF94. J Comput Chem. 1996;17: 490-519.

33. HyperChem. Professional version 8.0. Gainesville, FL, USA; Hypercube Inc; 2007.

34. Jorgensen WL, Maxwell DS, Tirado-Rives J. Development and testing of the OPLS all-atom force field on conformational energetics and properties of organic liquids. J Am Chem Soc. 1996;118:11225-11236.

35. Allinger NL. Conformational analysis. 130. MM2. A hydrocarbon force field utilizing V1 and V2 torsional terms. J Am Chem Soc. 1977;99: 8127-8134.

36. Fletcher R, Reeves CM. Function minimization by conjugate gradients. Comput J. 1964;7:149-154.

37. Brooks BR, Bruccoleri RE, Olafson BD, et al. CHARMM: A program for macromolecular energy, minimization, and dynamics calculations J Comput Chem. 1983;4:187-217.

38. Brooks BR, Brooks CL, Mackerell AD, et al. CHARMM: the biomolecular simulation program. J Comput Chem. 2009;30:1545-1614.

39. Ping $\mathrm{QN}$, Zhang $\mathrm{C}, \mathrm{Ye} \mathrm{H}$, et al. Polyethylene glycol prodrug of gambogic acid, its preparation method, formulation and use for preparing of drug. CN Patent No 100531801 C, Aug 26, 2009.

40. Tang N, Du G, Wang N, et al. Improving penetration in tumors with nanoassemblies of phospholipids and doxorubicin. J Natl Cancer Inst. 2007;99:1004-1015.

41. Liu J, Xiao Y, Allen C. Polymer-drug compatibility: a guide to the development of delivery systems for the anticancer agent, ellipticine. J Pharm Sci. 2004;93:132-143.
42. Liu J, Zahedi P, Zeng F, et al. Nano-sized assemblies of a PEG-docetaxel conjugate as a formulation strategy for docetaxel. J Pharm Sci. 2008;97: $3274-3290$

43. Lundberg BB, Risovic V, Ramaswamy M, et al. A lipophilic paclitaxel derivative incorporated in a lipid emulsion for parenteral administration. J Control Release. 2003;86:93-100.

44. Gabizon A, Horowitz AT, Goren D, et al. Targeting folate receptor with folate linked to extremities of poly (ethylene glycol)-grafted liposomes: in vitro studies. Bioconjug Chem. 1999;10:289-298.

45. Lee RJ, Low PS. Folate-mediated tumor cell targeting of liposomeentrapped doxorubicin in vitro. Biochim Biophys Acta. 1995;1233: 134-144.

46. Greenwald RB. PEG drugs: an overview. J Control Release. 2001;74: 159-171.

47. Minko T. Soluble polymer conjugates for drug delivery. Drug Discov Today. 2005;2:15-20.

48. Langer CJ. CT-2103: a novel macromolecular taxane with potential advantages compared with conventional taxanes. Clin Lung Cancer. 2004;6:S85-S88.

49. Vasey PA, Kaye SB, Morrison R, et al. Phase I clinical and pharmacokinetic study of PK1 [N-(2-hydroxypropyl)methacrylamide copolymer doxorubicin]: first member of a new class of chemotherapeutic agents-drug-polymer conjugates. Cancer Research Campaign Phase I/II Committee. Clin Cancer Res. 1999;5:83-94.

50. Seymour LW, Ferry DR, Anderson D, et al. Hepatic drug targeting: Phase I evaluation of polymer-bound doxorubicin. $J$ Clin Oncol. 2002;20:1668-1676.

51. Meerum Terwogt JM, ten Bokkel Huinink WW, Schellens JH, et al. Phase I clinical and pharmacokinetic study of PNU166945, a novel water-soluble polymer-conjugated prodrug of paclitaxel. Anticancer Drugs. 2001;12:315-323.

52. Schoemaker NE, van Kesteren C, Rosing H, et al. A phase I and pharmacokinetic study of MAG-CPT, a water-soluble polymer conjugate of camptothecin. Br J Cancer. 2002;87:608-614.

53. Tofovic SP, Jackson EK. Effects of long-term caffeine consumption on renal function in spontaneously hypertensive heart failure prone rats. J Cardiovasc Pharmacol. 1999;33:360-366.

54. Stoyanova E, Mitova V, Shestakova P, et al. Reversibly PEGylated nanocarrier for cisplatin delivery. J Inorg Biochem. 2013;120: $54-62$.
International Journal of Nanomedicine

\section{Publish your work in this journal}

The International Journal of Nanomedicine is an international, peerreviewed journal focusing on the application of nanotechnology in diagnostics, therapeutics, and drug delivery systems throughout the biomedical field. This journal is indexed on PubMed Central,

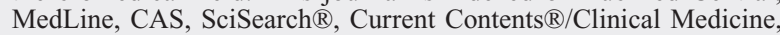

\section{Dovepress}

Journal Citation Reports/Science Edition, EMBase, Scopus and the Elsevier Bibliographic databases. The manuscript management system is completely online and includes a very quick and fair peer-review system, which is all easy to use. Visit http://www.dovepress.com/ testimonials.php to read real quotes from published authors. 